\title{
Hindered aryl bromides for regioselective palladium- catalysed direct arylation at less favourable C5-carbon of 3-substituted thiophenes
}

\author{
Rongwei Jin, Charles Beromeo Bheeter and Henri Doucet ${ }^{*}$
}

\section{Letter}

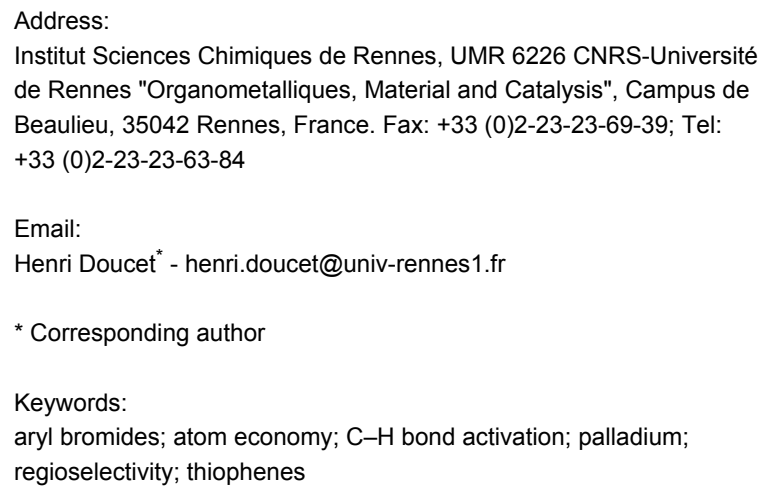

\author{
Beilstein J. Org. Chem. 2014, 10, 1239-1245. \\ doi:10.3762/bjoc. 10.123 \\ Received: 24 February 2014 \\ Accepted: 24 April 2014 \\ Published: 27 May 2014 \\ Associate Editor: I. Marek \\ (C) 2014 Jin et al; licensee Beilstein-Institut. \\ License and terms: see end of document.
}

\begin{abstract}
The use of the congested aryl bromide 2-bromo-1,3-dichlorobenzene as coupling partner allows to modify the regioselectivity of the arylation of 3-substituted thiophene derivatives in favour of carbon C5. The coupling of this aryl bromide with a variety of 3-substituted thiophenes gave in all cases the desired 5-arylation products in moderate to good yields using only 0.5 mol \% of a phosphinefree and air-stable palladium catalyst. Then, from these 5-arylthiophenes, a second palladium-catalysed $\mathrm{C}-\mathrm{H}$ bond functionalization at $\mathrm{C} 2$ of the thiophene ring allows the synthesis of 2,5-diarylthiophenes with two different aryl units.
\end{abstract}

\section{Introduction}

As thiophenes bearing aryl substituents are known to be present in several bioactive molecules and are used as precursors of materials, the regioselective introduction of aryls on thiophenes is an important research area in organic synthesis. The coupling of thiophene derivatives with aryl halides via a $\mathrm{C}-\mathrm{H}$ bond activation/functionalisation [1-12] provides an environmentally attractive and cost-effective procedure for the preparation of a variety of arylated thiophenes. For such coupling reactions, the major byproduct is a base associated to HX, instead of metallic salts which are produced under the more classical Negishi,
Suzuki or Stille cross-coupling reactions. Moreover, direct arylation avoids the preliminary preparation of organometallics reducing the number of steps to prepare these arylthiophenes.

The regioselective arylation via a $\mathrm{Pd}$-catalysed $\mathrm{C}-\mathrm{H}$ bond activation at carbon $\mathrm{C} 5$ of 2 -substituted thiophenes has been largely described in recent years [13-22]. On the other hand, the Pd-catalysed direct arylation of 3-substituted thiophenes has attracted much less attention (Scheme 1, top) [23-31]. With such thiophene derivatives, in most cases, either $\mathrm{C} 2$-arylated 
thiophenes or mixtures of $\mathrm{C} 2$ - and $\mathrm{C} 5$-arylated products have been obtained. For example, Sharp et al. described conditions for the regioselective arylation at carbons $\mathrm{C} 2$ or C5 of methyl 3-thiophenecarboxylate [23]. The reaction performed with $\mathrm{Pd}\left(\mathrm{PPh}_{3}\right)_{4}$ as the catalyst in toluene led selectively to the 2-arylated thiophene; whereas, the use of $\operatorname{Pd}_{2}(\mathrm{dba})_{3}$ in NMP afforded a mixture of 2- and 5-arylated thiophenes in a 15:51 ratio. Lemaire and co-workers have reported the $\mathrm{C} 2$-arylation of 3-formyl-, 3-cyano- and 3-nitrothiophenes with iodobenzenes $[24,25]$. Forgione, Bilodeau et al. reported that the reaction of 3 -methylthiophene with bromobenzene using $\operatorname{Pd}\left[\left(\mathrm{P}(t-\mathrm{Bu})_{3}\right]_{2}\right.$ as the catalyst affords a mixture of 2- and 5-phenylated thiophenes in a 3.3:1 ratio [26]. Fagnou and co-workers reported that the coupling of 3-n-hexylthiophene with 4-bromonitrobenzene also led to a mixture of $\mathrm{C} 2$ - and $\mathrm{C} 5$-arylation products in a 1.3:1 ratio [27]. Then, they blocked one position on the thiophene ring using a chloro-substituent in order to selectively arylate positions $\mathrm{C} 2$ or $\mathrm{C} 5$. The direct arylation of 3-methoxythiophene, which was studied by Borghese and co-workers afforded regioselectively the $\mathrm{C} 2$-arylated thiophenes in moderate to high yields [28]. Finally, in the course of their studies on $\mathrm{sp}^{3} \mathrm{C}-\mathrm{H}$ bond activation, Baudoin and Pierre recently reported that in the presence of an extremely bulky substituent at $\mathrm{C} 3$ of a thiophene derivative, the C5-arylated compounds were selectively obtained in good yields [29]. In summary, due to the presence of two reactive $\mathrm{C}-\mathrm{H}$ bonds in 3 -substituted thiophenes (with position $\mathrm{C} 2$ generally slightly more reactive than position $\mathrm{C} 5$ ), the control of the regioselectivity of the palladium-catalysed direct arylation of such thiophene derivatives especially to provide 5-arylthiophenes remains a challenging reaction.

Our goal was to promote arylation at carbon C5 of a range of 3 -substituted thiophenes without the use of a blocking group at carbon C2. To our knowledge, ortho-substituents on aryl bromides have not been employed as directing groups for palladium-catalysed direct arylation of 3-substituted thiophenes. The use of congested aryl bromides for such couplings would certainly modify the regioselectivity in favour of arylation at the less hindered thiophene position. Herein, we wish to report on the influence of such ortho-substituents on aryl bromides on the regioselectivity of the palladium-catalysed direct arylations of 3 -substituted thiophenes.

\section{Results and Discussion}

We first studied the palladium-catalysed direct arylation of 3-methylthiophene using tert-butyl 2-bromobenzoate as the coupling partner (Scheme 2). Based on previous results [19], DMA was initially chosen as the solvent and KOAc as the base. The reactions were performed at $150{ }^{\circ} \mathrm{C}$ under argon in the presence of $0.5 \mathrm{~mol} \% \mathrm{Pd}(\mathrm{OAc})_{2}$ as the catalyst. However, under these conditions, a poor regioselectivity was observed as the desired C5-arylation product $\mathbf{1 b}$ was only obtained in 34\% selectivity together with $66 \%$ of $\mathrm{C} 2$-arylation product $1 \mathrm{a}$. Moreover, a moderate conversion of this aryl bromide was observed and purification by silica gel chromatography afforded a mixture of 1a and 1b. A slightly lower selectivity in favour of the formation of $\mathbf{C 5}$-arylation product $\mathbf{2} \mathbf{b}$ was observed using

\section{Previous work}
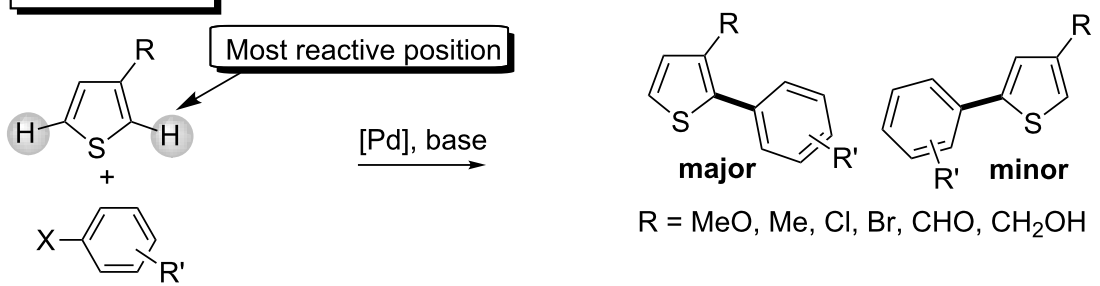

$\mathrm{R}=\mathrm{MeO}, \mathrm{Me}, \mathrm{Cl}, \mathrm{Br}, \mathrm{CHO}, \mathrm{CH}_{2} \mathrm{OH}$

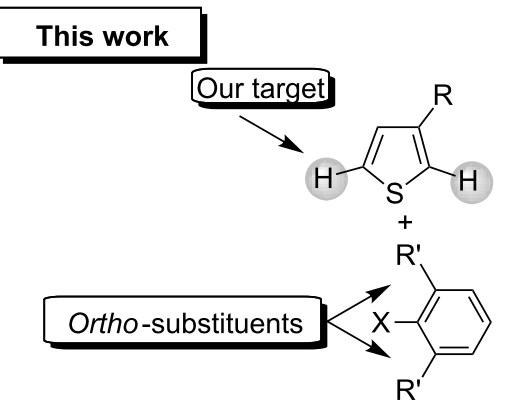

[Pd], base

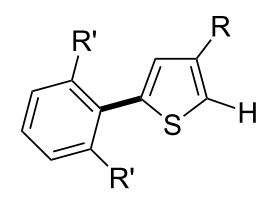




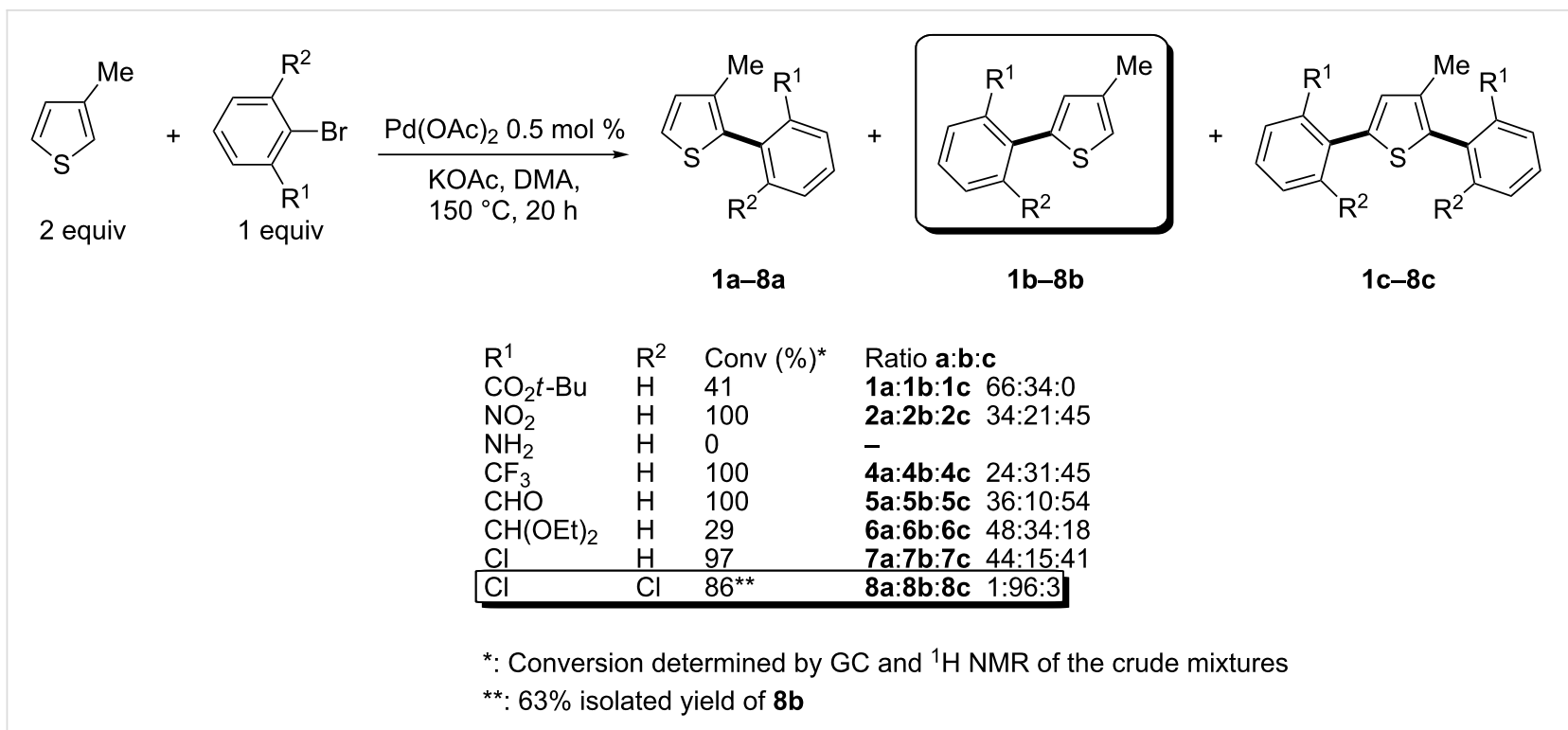

Scheme 2: Regioselectivity of the arylation of 2-methylthiophene with ortho-substituted aryl bromides.

2-bromonitrobenzene as the coupling partner, as a large amount of 2,5-diarylated product $2 \mathbf{c}$ was also produced with a $2 \mathbf{a}: \mathbf{2 b}: \mathbf{2} \mathbf{c}$ ratio of 34:21:45. However, a complete conversion of 2-bromonitrobenzene was observed. 2-Bromoaniline was found to be unreactive and was recovered. It should be noted that no amination reaction of 2-bromoaniline due to self-coupling was observed. From 2-(trifluoromethyl)bromobenzene, a very similar mixture of regioisomers than with 2-bromonitrobenzene was obtained, as $\mathbf{4 a}, \mathbf{4 b}$ and $\mathbf{4 c}$ were formed in a 24:31:45 ratio. The use of 2-bromobenzaldehyde was not successful, as the desired product $\mathbf{5 b}$ was only obtained with $10 \%$ selectivity. From more hindered 2-bromobenzaldehyde diethyl acetal, the selectivity in favour of desired product $\mathbf{6 b}$ was slightly higher (34\%), although still not synthetically useful. Next, we employed 2-chlorobromobenzene as the coupling partner. However, again the desired product $\mathbf{7 b}$ was only formed with $15 \%$ selectivity. It should be noted that with these aryl bromides, in all cases, mixtures of regioisomers $\mathbf{a}$ and $\mathbf{b}$ were obtained after column chromatography. On the other hand, the use of 2-bromo-1,3-dichlorobenzene allowed to obtain very selectively the desired 5-arylation product $\mathbf{8 b}$. Only traces of the C2-arylated thiophene $8 \mathbf{a}$ and a low amount of 2,5-diarylated product $8 \mathbf{c}$ were detected by ${ }^{1} \mathrm{H}$ NMR and GC-MS analysis of the crude mixture. Moreover a high conversion of $86 \%$ of this aryl bromide was observed using only $0.5 \mathrm{~mol} \% \mathrm{Pd}(\mathrm{OAc})_{2}$ as the catalyst.

Then, we studied the scope of the coupling of 2-bromo-1,3dichlorobenzene, using other 3-substituted thiophene derivatives (Scheme 3, Table 1). Both 3-chlorothiophene and ethyl thiophene-3-carboxylate led to the desired 5-arylation products 9b and 10b with $89 \%$ selectivity and in $44 \%$ and $65 \%$ yields, respectively (Table 1, entries 1 and 2). A slightly higher regioselectivity in favour of the C5-arylation was observed in the presence of 3-acetylthiophene, as $\mathbf{1 1 b}$ was obtained with $92 \%$ selectivity (Table 1, entry 3). From ethyl thiophen-3-ylacetate, 12b was formed with $90 \%$ selectivity and in $62 \%$ yield (Table 1, entry 4). On the other hand, from methyl (E)-3-thiophen-3-ylacrylate, 3-bromothiophene or 3-formylthiophene, mixtures of unidentified products were obtained.

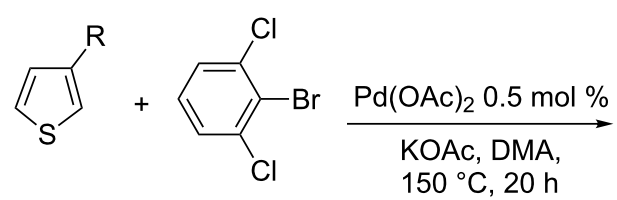

$150^{\circ} \mathrm{C}, 20 \mathrm{~h}$<smiles>[R]c1ccsc1-c1c(Cl)cccc1Cl</smiles>

a

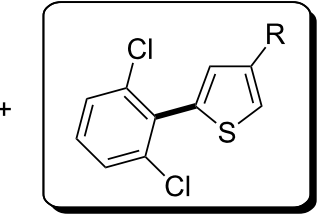

$9 b-12 b$<smiles>[R]c1cc(-c2c(Cl)cc[cH+]cc2Cl)sc1-c1c(Cl)cccc1Cl</smiles>

c 


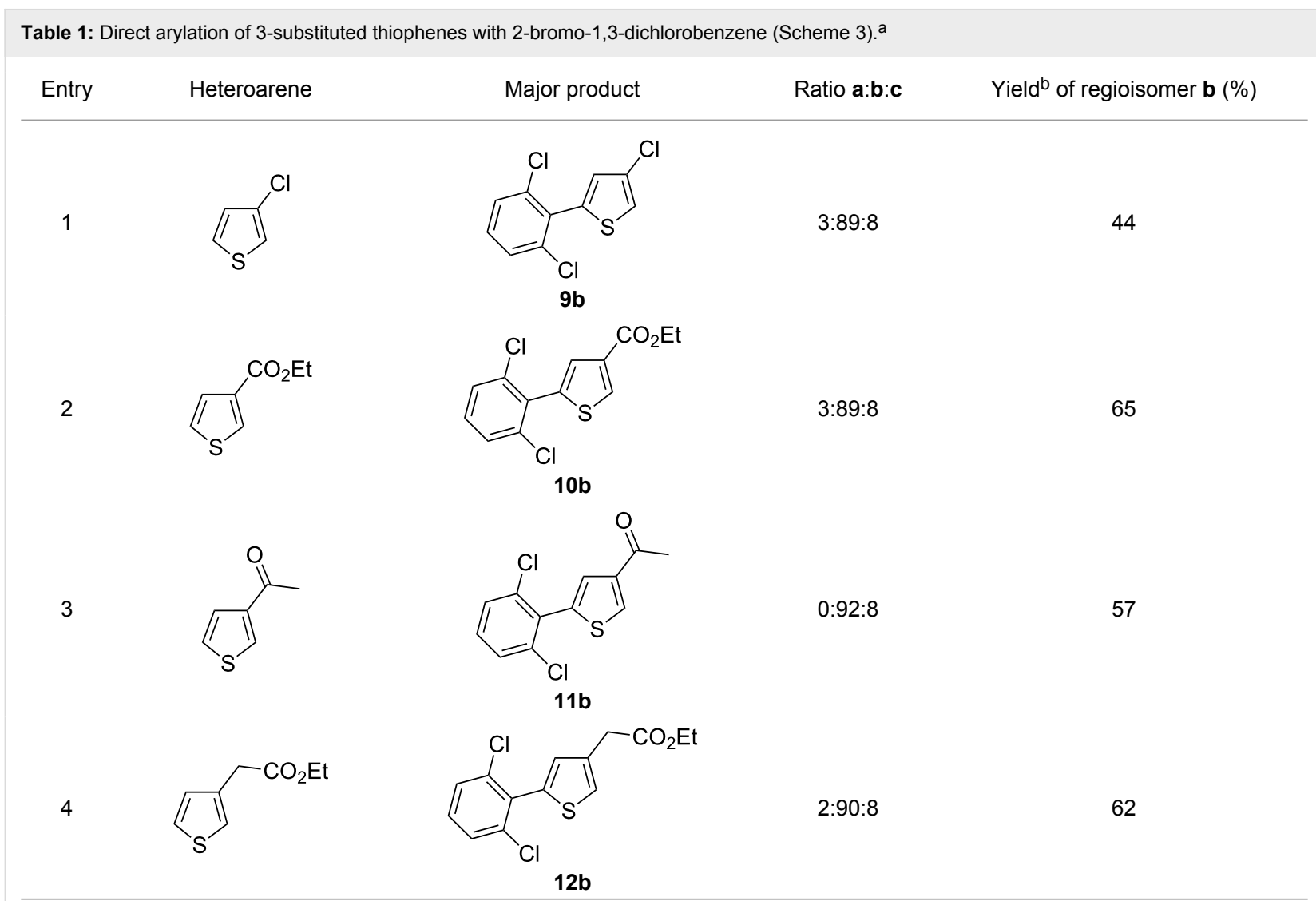

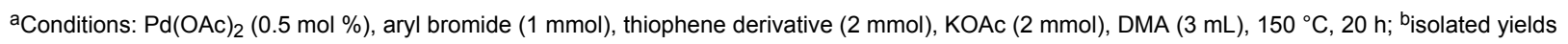
of regioisomers $\mathbf{9 b}-\mathbf{1 2 b}$.

The reactivity for arylation at carbon $\mathrm{C} 2$ of the previously prepared 2-(2,6-dichlorophenyl)-4-methylthiophene (8b), via a second Pd-catalysed $\mathrm{C}-\mathrm{H}$ bond activation, was also investigated (Scheme 4). 4-Bromobenzonitrile and $\mathbf{8 b}$ in the presence of $0.5 \mathrm{~mol} \% \mathrm{Pd}(\mathrm{OAc})_{2}$ and $\mathrm{KOAc}$ as the base gave the desired coupling product $\mathbf{1 3}$ in $82 \%$ yield. A similar reactivity was observed with 4-bromobenzaldehyde and 3-bromoacetophenone affording 14 and 15 in $80 \%$ and $85 \%$ yields, respectively. Finally, more congested 2-bromobenzonitrile was reacted with $\mathbf{8 b}$ to provide $\mathbf{1 6}$ in $88 \%$ yield. These regioselective sequential arylations of a 3-substituted thiophene offer a simple access to a variety of 2,5-diarylthiophenes with two different aryl units.

\section{Conclusion}

In summary, we have demonstrated that the use of the congested coupling partner 2-bromo-1,3-dichlorobenzene allows to direct the arylation to the unfavourable $\mathrm{C} 5$ position of 3 -substituted thiophenes. These less favoured regioisomers can be selectively obtained in moderate to good yields using a range of 3-substituted thiophenes, as chloro, ester, acetyl or ethyl<smiles>Cc1csc(-c2c(Cl)cccc2Cl)c1</smiles>

$8 b$

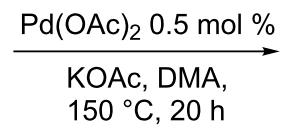

$150^{\circ} \mathrm{C}, 20 \mathrm{~h}$<smiles>[R]c1ccccc1-c1sc(-c2c(Cl)cccc2Cl)cc1C</smiles>

$$
\begin{array}{lll}
\mathrm{R} & & \text { Yield (\%) } \\
\text { 4-CN } & \mathbf{1 3} & 82 \\
\text { 4-CHO } & \mathbf{1 4} & 80 \\
\text { 3-COMe } & \mathbf{1 5} & 85 \\
\text { 2-CN } & \mathbf{1 6} & 88
\end{array}
$$


acetate substituents are tolerated. Moreover, the sequential catalytic C5 and C2 arylations, allow the preparation of 2,5diarylthiophenes with two different aryl units in two steps. The major byproduct of these couplings is $\mathrm{KBr} / \mathrm{AcOH}$ instead of metallic salts as with more classical coupling procedures. For these reasons, this process gives an economically viable access to C5-arylated 3-substituted heteroaromatics.

\section{Experimental General}

All reactions were perfomed in Schlenk tubes under argon. DMA analytical grade was not distilled before use. Commercial aryl bromide derivatives were used without purification. ${ }^{1} \mathrm{H}$ NMR (400 MHz), ${ }^{13} \mathrm{C}$ NMR $(100 \mathrm{MHz})$ spectra were recorded in $\mathrm{CDCl}_{3}$ solutions on Bruker GPX (400 MHz). GC was performed on a Shimadzu GC-2014 and GC-MS was performed on a Shimadzu QP-2010S. Chemical shifts are reported in ppm relative to $\mathrm{CDCl}_{3}\left({ }^{1} \mathrm{H}: 7.26\right.$ and $\left.{ }^{13} \mathrm{C}: 77.0\right)$. Flash chromatography was performed on silica gel (230-400 mesh).

\section{General procedure for direct arylations}

The aryl bromide $(1 \mathrm{mmol})$, thiophene derivative $(2 \mathrm{mmol})$, KOAc (2 mmol), Pd(OAc) $)_{2}(0.005 \mathrm{mmol}, 1.1 \mathrm{mg})$ and DMA $(3 \mathrm{~mL})$ were introduced in a Schlenk tube under argon equipped with a magnetic stirring bar. The Schlenk tube was placed in an oil bath pre-heated at $150{ }^{\circ} \mathrm{C}$, and the reaction mixture was allowed to stir for $20 \mathrm{~h}$. After cooling, the crude reaction mixture was analysed by gas chromatography and ${ }^{1} \mathrm{H}$ NMR to determine the conversion of the aryl bromide and the regioselectivity of the arylation. The solvent was removed by heating under vacuum, then the residue was charged onto a silica gel column.

tert-Butyl 2-(4-methylthiophen-2-yl)benzoate (1b): After column chromatography (pentane/diethyl ether 98:2), a mixture of products 1a and 1b was obtained in 24\% (0.066 g) yield. ${ }^{1} \mathrm{H} \mathrm{NMR}\left(400 \mathrm{MHz}, \mathrm{CDCl}_{3}\right) \delta 6.84(\mathrm{~s}, 1 \mathrm{H}), 2.20(\mathrm{~s}, 3 \mathrm{H}) .1 \mathbf{a}$ was also observed: ${ }^{1} \mathrm{H}$ NMR $\left(400 \mathrm{MHz}, \mathrm{CDCl}_{3}\right) \delta 7.14(\mathrm{~d}, J=$ $5.0 \mathrm{~Hz}, 1 \mathrm{H}), 6.81(\mathrm{~d}, J=5.0 \mathrm{~Hz}, 1 \mathrm{H}), 1.97(\mathrm{~s}, 3 \mathrm{H})$.

4-Methyl-2-(2-nitrophenyl)thiophene (2b): After column chromatography (pentane/diethyl ether 95:5), a mixture of products $2 \mathbf{a}$ and $\mathbf{2 b}$ was obtained in $60 \%(0.131 \mathrm{~g})$ yield. ${ }^{1} \mathrm{H}$ NMR $\left(400 \mathrm{MHz}, \mathrm{CDCl}_{3}\right) \delta 7.63(\mathrm{~d}, J=8.0 \mathrm{~Hz}, 1 \mathrm{H}), 6.91(\mathrm{~s}, 1 \mathrm{H}), 2.19$ (s, 3H). 2a was also observed: ${ }^{1} \mathrm{H}$ NMR $\left(400 \mathrm{MHz}, \mathrm{CDCl}_{3}\right) \delta$ $7.83(\mathrm{~d}, J=8.0 \mathrm{~Hz}, 1 \mathrm{H}), 7.22(\mathrm{~d}, J=5.0 \mathrm{~Hz}, 1 \mathrm{H}), 6.83(\mathrm{~d}, J=$ $5.0 \mathrm{~Hz}, 1 \mathrm{H}), 1.99$ (s, 3H).

4-Methyl-2-(2-trifluoromethylphenyl)thiophene (4b): After column chromatography (pentane/diethyl ether 90:10), a mix- ture of products $4 \mathbf{a}, \mathbf{4 b}$ and $\mathbf{4 c}$ was obtained in $66 \%(0.160 \mathrm{~g})$ yield. ${ }^{1} \mathrm{H}$ NMR $\left(400 \mathrm{MHz}, \mathrm{CDCl}_{3}\right) \delta 6.88(\mathrm{~s}, 1 \mathrm{H}), 6.83(\mathrm{~s}, 1 \mathrm{H})$, $1.95(\mathrm{~s}, 3 \mathrm{H}) .4 \mathrm{a}$ was also observed: ${ }^{1} \mathrm{H}$ NMR $(400 \mathrm{MHz}$, $\left.\mathrm{CDCl}_{3}\right) \delta 7.20(\mathrm{~d}, J=5.1 \mathrm{~Hz}, 1 \mathrm{H}), 6.81(\mathrm{~d}, J=5.1 \mathrm{~Hz}, 1 \mathrm{H}) .4 \mathrm{c}$ was also observed: ${ }^{1} \mathrm{H}$ NMR $\left(400 \mathrm{MHz}, \mathrm{CDCl}_{3}\right) \delta 7.75-7.65$ $(\mathrm{m}, 2 \mathrm{H}), 7.55-7.35(\mathrm{~m}, 6 \mathrm{H}), 6.82(\mathrm{~s}, 1 \mathrm{H}), 1.95(\mathrm{~s}, 3 \mathrm{H})$.

2-(4-Methylthiophen-2-yl)benzaldehyde (5b): After column chromatography (pentane/diethyl ether 95:5), a mixture of products $5 \mathbf{a}$ and $\mathbf{5 b}$ was obtained in 51\% (0.103 g) yield. ${ }^{1} \mathrm{H}$ NMR $\left(400 \mathrm{MHz}, \mathrm{CDCl}_{3}\right) \delta 10.14(\mathrm{~s}, 1 \mathrm{H}), 7.92(\mathrm{~d}, J=7.8 \mathrm{~Hz}, 1 \mathrm{H})$, $7.60-7.35(\mathrm{~m}, 3 \mathrm{H}), 6.97(\mathrm{~s}, 1 \mathrm{H}), 6.81(\mathrm{~s}, 1 \mathrm{H}), 2.25(\mathrm{~s}, 3 \mathrm{H}) .5 \mathbf{a}$ was also observed: ${ }^{1} \mathrm{H}$ NMR $\left(400 \mathrm{MHz}, \mathrm{CDCl}_{3}\right) \delta 9.85(\mathrm{~s}, 1 \mathrm{H})$, $7.95(\mathrm{~d}, J=7.6 \mathrm{~Hz}, 1 \mathrm{H}), 7.56(\mathrm{t}, J=7.6 \mathrm{~Hz}, 1 \mathrm{H}), 7.44(\mathrm{t}, J=7.6$ $\mathrm{Hz}, 1 \mathrm{H}), 7.36(\mathrm{~d}, J=7.6 \mathrm{~Hz}, 1 \mathrm{H}), 7.27(\mathrm{~d}, J=5.0 \mathrm{~Hz}, 1 \mathrm{H}), 6.89$ $(\mathrm{d}, J=5.0 \mathrm{~Hz}, 1 \mathrm{H}), 2.02(\mathrm{~s}, 3 \mathrm{H})$.

2-(2-Chlorophenyl)-4-methylthiophene (7b): After column chromatography (pentane/diethyl ether 95:5), a mixture of products $7 \mathbf{a}$ and $7 \mathbf{b}$ was obtained in $64 \%(0.133 \mathrm{~g})$ yield. ${ }^{1} \mathrm{H}$ NMR $\left(400 \mathrm{MHz}, \mathrm{CDCl}_{3}\right) \delta 7.11(\mathrm{~s}, 1 \mathrm{H}), 6.90(\mathrm{~s}, 1 \mathrm{H}), 2.24(\mathrm{~s}, 3 \mathrm{H}) .7 \mathbf{a}$ was also observed: ${ }^{1} \mathrm{H} \mathrm{NMR}\left(400 \mathrm{MHz}, \mathrm{CDCl}_{3}\right) \delta 6.87(\mathrm{~d}, J=$ $5.0 \mathrm{~Hz}, 1 \mathrm{H}), 2.04(\mathrm{~s}, 3 \mathrm{H})$.

2-(2,6-Dichlorophenyl)-4-methylthiophene (8b): From 2-bromo-1,3-dichlorobenzene $(0.226 \mathrm{~g}, 1 \mathrm{mmol})$ and 3-methylthiophene (0.196 g, $2 \mathrm{mmol}), \mathbf{8 b}$ was obtained in 63\% (0.153 g) yield as an oil after column chromatography (pentane). ${ }^{1} \mathrm{H}$ NMR (400 MHz, $\left.\mathrm{CDCl}_{3}\right) \delta 7.31(\mathrm{~d}, J=7.5 \mathrm{~Hz}, 2 \mathrm{H}), 7.15(\mathrm{t}$, $J=7.5 \mathrm{~Hz}, 1 \mathrm{H}), 6.98(\mathrm{~s}, 1 \mathrm{H}), 6.75(\mathrm{~s}, 1 \mathrm{H}), 2.25(\mathrm{~s}, 3 \mathrm{H}) ;{ }^{13} \mathrm{C}$ NMR $\left(50 \mathrm{MHz}, \mathrm{CDCl}_{3}\right) \delta 137.4,136.6,136.4,133.1,130.8$, 129.7, 128.0, 122.1, 15.8; Anal. calcd for $\mathrm{C}_{11} \mathrm{H}_{8} \mathrm{Cl}_{2} \mathrm{~S}$ (243.15): C, 54.34; H, 3.32; found: C, 54.19; H, 3.17. Traces of $\mathbf{8 a}$ and $\mathbf{8 c}$ were also detected by GC-MS analysis of the crude mixture.

4-Chloro-2-(2,6-dichlorophenyl)thiophene (9b): From 2-bromo-1,3-dichlorobenzene $(0.226 \mathrm{~g}, 1 \mathrm{mmol})$ and 3-chlorothiophene $(0.237 \mathrm{~g}, 2 \mathrm{mmol})$, 9b was obtained in 44\% (0.116 g) yield as an oil after column chromatography (pentane). ${ }^{1} \mathrm{H}$ NMR (400 MHz, $\left.\mathrm{CDCl}_{3}\right) \delta 7.32(\mathrm{~d}, J=7.5 \mathrm{~Hz}, 2 \mathrm{H})$, $7.23-7.17(\mathrm{~m}, 2 \mathrm{H}), 6.83(\mathrm{~d}, J=1.2 \mathrm{~Hz}, 1 \mathrm{H}) ;{ }^{13} \mathrm{C}$ NMR $(50$ $\left.\mathrm{MHz}, \mathrm{CDCl}_{3}\right) \delta 137.4,136.5,131.7,130.4,128.8,128.2,124.9$, 121.4; Anal. calcd for $\mathrm{C}_{10} \mathrm{H}_{5} \mathrm{Cl}_{3} \mathrm{~S}$ (263.57): $\mathrm{C}, 45.57$; $\mathrm{H}, 1.91$; found: $\mathrm{C}, 45.67 ; \mathrm{H}, 1.90$. Traces of $9 \mathrm{a}$ and $9 \mathrm{c}$ were also detected by GC-MS analysis of the crude mixture.

Ethyl 5-(2,6-dichlorophenyl)thiophene-3-carboxylate (10b): From 2-bromo-1,3-dichlorobenzene $(0.226 \mathrm{~g}, 1 \mathrm{mmol})$ and ethyl thiophene-3-carboxylate $(0.312 \mathrm{~g}, 2 \mathrm{mmol}), \mathbf{1 0 b}$ was obtained in $65 \%(0.196 \mathrm{~g})$ yield as an oil after column chromatography (pentane/diethyl ether 98:2). ${ }^{1} \mathrm{H}$ NMR (400 MHz, 
$\left.\mathrm{CDCl}_{3}\right) \delta 8.16(\mathrm{~s}, 1 \mathrm{H}), 7.37(\mathrm{~d}, J=7.5 \mathrm{~Hz}, 2 \mathrm{H}), 7.34(\mathrm{~s}, 1 \mathrm{H})$, $7.22(\mathrm{t}, J=7.5 \mathrm{~Hz}, 1 \mathrm{H}), 4.29$ (q, $J=7.5 \mathrm{~Hz}, 2 \mathrm{H}), 1.31$ (t, $J=7.5$ $\mathrm{Hz}, 3 \mathrm{H}) ;{ }^{13} \mathrm{C} \mathrm{NMR}\left(50 \mathrm{MHz}, \mathrm{CDCl}_{3}\right) \delta 162.6,137.3,136.6$, 133.8, 133.7, 131.8, 130.3, 129.3, 128.2, 60.8, 14.3; Anal. calcd for $\mathrm{C}_{13} \mathrm{H}_{10} \mathrm{Cl}_{2} \mathrm{O}_{2} \mathrm{~S}$ (301.19): C, 51.84; $\mathrm{H}, 3.35$; found: $\mathrm{C}, 51.99$; $\mathrm{H}, 3.17$. Traces of $\mathbf{1 0 a}$ and $\mathbf{1 0 c}$ were also detected by GC-MS analysis of the crude mixture.

1-[5-(2,6-Dichlorophenyl)thiophen-3-yl] ethanone (11b): From 2-bromo-1,3-dichlorobenzene $(0.226 \mathrm{~g}, 1 \mathrm{mmol})$ and 1-thiophen-3-ylethanone (0.252 g, $2 \mathrm{mmol})$, 11b was obtained in $57 \%(0.154 \mathrm{~g})$ yield as an oil after column chromatography (pentane/diethyl ether 95:5). ${ }^{1} \mathrm{H}$ NMR $\left(400 \mathrm{MHz}, \mathrm{CDCl}_{3}\right) \delta$ $8.10(\mathrm{~s}, 1 \mathrm{H}), 7.37(\mathrm{~d}, J=7.5 \mathrm{~Hz}, 2 \mathrm{H}), 7.34(\mathrm{~s}, 1 \mathrm{H}), 7.22$ (t, $J=$ $7.5 \mathrm{~Hz}, 1 \mathrm{H}), 2.49$ (s, 3H); ${ }^{13} \mathrm{C} \mathrm{NMR}\left(50 \mathrm{MHz}, \mathrm{CDCl}_{3}\right) \delta 191.0$, 141.3, 136.7, 135.4, 132.5, 130.6, 129.4, 127.4, 127.2, 26.4; Anal. calcd (\%) for $\mathrm{C}_{12} \mathrm{H}_{8} \mathrm{Cl}_{2} \mathrm{OS}$ (271.16): C, 53.15; H, 2.97; found: $\mathrm{C}, 53.31 ; \mathrm{H}, 3.07$. Traces of $11 \mathrm{c}$ were also detected by GC-MS analysis of the crude mixture.

Ethyl [5-(2,6-dichlorophenyl)thiophen-3-yl]acetate (12b): From 2-bromo-1,3-dichlorobenzene $(0.226 \mathrm{~g}, 1 \mathrm{mmol})$ and ethyl thiophen-3-ylacetate $(0.340 \mathrm{~g}, 2 \mathrm{mmol}), \mathbf{1 2 b}$ was obtained in $62 \%(0.195 \mathrm{~g})$ yield as an oil after column chromatography (pentane/diethyl ether 95:5). ${ }^{1} \mathrm{H}$ NMR (400 $\left.\mathrm{MHz}, \mathrm{CDCl}_{3}\right) \delta$ $7.32(\mathrm{~d}, J=7.5 \mathrm{~Hz}, 2 \mathrm{H}), 7.22(\mathrm{~s}, 1 \mathrm{H}), 7.16(\mathrm{t}, J=7.5 \mathrm{~Hz}, 1 \mathrm{H})$, $6.89(\mathrm{~s}, 1 \mathrm{H}), 4.12(\mathrm{q}, J=7.5 \mathrm{~Hz}, 2 \mathrm{H}), 3.60(\mathrm{~s}, 2 \mathrm{H}), 1.19(\mathrm{t}, J=$ $7.5 \mathrm{~Hz}, 3 \mathrm{H}) ;{ }^{13} \mathrm{C} \mathrm{NMR}\left(50 \mathrm{MHz}, \mathrm{CDCl}_{3}\right) \delta 170.9,136.7,136.6$, 133.5, 132.8, 130.2, 129.9, 128.1, 124.2, 60.9, 36.2, 14.2; Anal. calcd for $\mathrm{C}_{14} \mathrm{H}_{12} \mathrm{Cl}_{2} \mathrm{O}_{2} \mathrm{~S}$ (315.22): C, 53.34; H, 3.84; found: $\mathrm{C}$, 53.21; H, 3.70. Traces of $12 \mathbf{a}$ and $12 \mathbf{c}$ were also detected by GC-MS analysis of the crude mixture.

4-[5-(2,6-Dichlorophenyl)-3-methylthiophen-2-yl]benzonitrile (13): From 4-bromobenzonitrile $(0.182 \mathrm{~g}, 1 \mathrm{mmol})$ and 2-(2,6-dichlorophenyl)-4-methylthiophene $(\mathbf{8 b}, 0.486 \mathrm{~g}$, $2 \mathrm{mmol}), 13$ was obtained in $82 \%(0.282 \mathrm{~g})$ yield as an oil after column chromatography (pentane/diethyl ether 95:5). ${ }^{1} \mathrm{H}$ NMR $\left(400 \mathrm{MHz}, \mathrm{CDCl}_{3}\right) \delta 7.63(\mathrm{~d}, J=7.5 \mathrm{~Hz}, 2 \mathrm{H}), 7.56(\mathrm{~d}, J=7.5$ $\mathrm{Hz}, 2 \mathrm{H}), 7.34$ (d, $J=7.5 \mathrm{~Hz}, 2 \mathrm{H}), 7.19$ (t, $J=7.5 \mathrm{~Hz}, 1 \mathrm{H}), 6.80$ $(\mathrm{s}, 1 \mathrm{H}), 2.33(\mathrm{~s}, 3 \mathrm{H}) ;{ }^{13} \mathrm{C} \mathrm{NMR}\left(50 \mathrm{MHz}, \mathrm{CDCl}_{3}\right) \delta 139.1$, 137.2, 136.4, 136.3, 134.7, 133.2, 132.3, 132.2, 130.0, 129.2, 128.2, 118.8, 110.6, 15.8; Anal. calcd for $\mathrm{C}_{18} \mathrm{H}_{11} \mathrm{Cl}_{2} \mathrm{NS}$ (344.26): C, 62.80; H, 3.22; found: C, 63.04; H, 3.17.

4-(5-(2,6-Dichlorophenyl)-3-methylthiophen-2-yl)benzaldehyde (14): From 4-bromobenzaldehyde $(0.185 \mathrm{~g}, 1 \mathrm{mmol})$ and 2-(2,6-dichlorophenyl)-4-methylthiophene $(\mathbf{8 b}, 0.486 \mathrm{~g}$, $2 \mathrm{mmol}), \mathbf{1 6}$ was obtained in $80 \%(0.278 \mathrm{~g})$ yield as an oil after column chromatography (pentane/diethyl ether 85:15). ${ }^{1} \mathrm{H}$ NMR (400 MHz, $\left.\mathrm{CDCl}_{3}\right) \delta 9.97(\mathrm{~s}, 1 \mathrm{H}), 7.86(\mathrm{~d}, J=7.5 \mathrm{~Hz}$,
2H), $7.63(\mathrm{~d}, J=7.5 \mathrm{~Hz}, 2 \mathrm{H}), 7.34(\mathrm{~d}, J=7.5 \mathrm{~Hz}, 2 \mathrm{H}), 7.19$ (t, $J$ $=7.5 \mathrm{~Hz}, 1 \mathrm{H}), 6.81(\mathrm{~s}, 1 \mathrm{H}), 2.36(\mathrm{~s}, 3 \mathrm{H}) ;{ }^{13} \mathrm{C} \mathrm{NMR}(50 \mathrm{MHz}$, $\left.\mathrm{CDCl}_{3}\right) \delta 191.6,140.6,137.9,136.4,136.1,134.8,134.6$, 133.2, 132.3, 130.0, 129.1, 128.2, 15.5; Anal. calcd for $\mathrm{C}_{18} \mathrm{H}_{12} \mathrm{Cl}_{2} \mathrm{OS}$ (347.26): C, 62.26; H, 3.48; found: $\mathrm{C}, 62.09 ; \mathrm{H}$, 3.50 .

1-\{3-[5-(2,6-Dichlorophenyl)-3-methylthiophen-2yl]phenyl\}ethanone (15): From 3-bromoacetophenone (0.199 g, $1 \mathrm{mmol})$ and 2-(2,6-dichlorophenyl)-4-methylthiophene (8b, $0.486 \mathrm{~g}, 2 \mathrm{mmol}), 15$ was obtained in $85 \%$ (0.307 g) yield as a white solid after column chromatography pentane/ diethyl ether 85:15). ${ }^{1} \mathrm{H}$ NMR (400 MHz, $\left.\mathrm{CDCl}_{3}\right) \delta 8.04(\mathrm{~s}$, $1 \mathrm{H}), 7.84(\mathrm{~d}, J=7.5 \mathrm{~Hz}, 1 \mathrm{H}), 7.65$ (d, $J=7.5 \mathrm{~Hz}, 1 \mathrm{H}), 7.45$ (t, $J$ $=7.5 \mathrm{~Hz}, 1 \mathrm{H}), 7.34(\mathrm{~d}, J=7.5 \mathrm{~Hz}, 2 \mathrm{H}), 7.17(\mathrm{t}, J=7.5 \mathrm{~Hz}, 1 \mathrm{H})$, $6.79(\mathrm{~s}, 1 \mathrm{H}), 2.58(\mathrm{~s}, 3 \mathrm{H}), 2.31(\mathrm{~s}, 3 \mathrm{H}) ;{ }^{13} \mathrm{C} \mathrm{NMR}(50 \mathrm{MHz}$, $\left.\mathrm{CDCl}_{3}\right) \delta 196.8,137.1,136.4,135.5,134.0,133.9,132.5$, 132.4, 131.7, 131.6, 128.8, 127.9, 127.8, 127.1, 126.1, 25.7, 14.1; Anal. calcd for $\mathrm{C}_{19} \mathrm{H}_{14} \mathrm{Cl}_{2} \mathrm{OS}$ (361.29): C, 63.19; $\mathrm{H}, 3.91$; found: C, 63.04; H, 3.99 .

2-[5-(2,6-Dichlorophenyl)-3-methylthiophen-2-yl]benzonitrile (16): From 2-bromobenzonitrile $(0.182 \mathrm{~g}, 1 \mathrm{mmol})$ and 2-(2,6-dichlorophenyl)-4-methylthiophene (8b, $0.486 \mathrm{~g}$, $2 \mathrm{mmol}), 16$ was obtained in $88 \%(0.303 \mathrm{~g})$ yield as an oil after column chromatography (pentane/diethyl ether 95:5). ${ }^{1} \mathrm{H}$ NMR $\left(400 \mathrm{MHz}, \mathrm{CDCl}_{3}\right) \delta 7.77(\mathrm{~d}, J=7.8 \mathrm{~Hz}, 1 \mathrm{H}), 7.63(\mathrm{t}, J=7.8$ $\mathrm{Hz}, 1 \mathrm{H}), 7.57$ (d, $J=7.8 \mathrm{~Hz}, 1 \mathrm{H}), 7.46(\mathrm{t}, J=7.8 \mathrm{~Hz}, 1 \mathrm{H}), 7.40$ $(\mathrm{d}, J=7.8 \mathrm{~Hz}, 2 \mathrm{H}), 7.24(\mathrm{t}, J=7.8 \mathrm{~Hz}, 1 \mathrm{H}), 6.88(\mathrm{~s}, 1 \mathrm{H}), 2.27$ $(\mathrm{s}, 3 \mathrm{H}) ;{ }^{13} \mathrm{C} \mathrm{NMR}\left(50 \mathrm{MHz}, \mathrm{CDCl}_{3}\right) \delta 136.9,135.7,135.6$, $135.1,133.5,132.4,131.4,131.3,130.9,130.8,128.9,127.2$, 127.1, 117.0, 112.8, 13.9; Anal. calcd for $\mathrm{C}_{18} \mathrm{H}_{11} \mathrm{Cl}_{2} \mathrm{NS}$ (344.26): C, 62.80; H, 3.22; found: C, 63.01; H, 3.40.

\section{Acknowledgements}

This research was supported by a CEFIPRA fellowship. We thank the Centre National de la Recherche Scientifique and "Rennes Metropole" for providing financial support.

\section{References}

1. Bellina, F.; Rossi, R. Tetrahedron 2009, 65, 10269. doi:10.1016/j.tet.2009.10.015

2. Satoh, T.; Miura, M. Chem. Lett. 2007, 36, 200. doi:10.1246/cl.2007.200

3. Li, B.-J.; Yang, S.-D.; Shi, Z.-J. Synlett 2008, 949. doi:10.1055/s-2008-1042907

4. Ackermann, L.; Vincente, R.; Kapdi, A. R. Angew. Chem., Int. Ed. 2009, 48, 9792. doi:10.1002/anie.200902996

5. Fischmeister, C.; Doucet, H. Green Chem. 2011, 13, 741. doi:10.1039/c0gc00885k

6. Ackermann, L. Chem. Rev. 2011, 111, 1315. doi:10.1021/cr100412j 
7. Kuhl, N.; Hopkinson, M. N.; Wencel-Delord, J.; Glorius, F. Angew. Chem., Int. Ed. 2012, 51, 10236. doi:10.1002/anie.201203269

8. Neufeldt, S. R.; Sanford, M. S. Acc. Chem. Res. 2012, 45, 936. doi:10.1021/ar300014f

9. Wencel-Delord, J.; Glorius, F. Nat. Chem. 2013, 5, 369. doi:10.1038/nchem.1607

10. Akita, Y.; Inoue, A.; Yamamoto, K.; Ohta, A.; Kurihara, T.; Shimizu, M. Heterocycles 1985, 23, 2327. doi:10.3987/R-1985-09-2327

11. Ohta, A.; Akita, Y.; Ohkuwa, T.; Chiba, M.; Fukunaga, R.; Miyafuji, A.; Nakata, T.; Tani, N.; Aoyagi, Y. Heterocycles 1990, 31, 1951. doi:10.3987/COM-90-5467

12. Aoyagi, Y.; Inoue, A.; Koizumi, I.; Hashimoto, R.; Tokunaga, K.; Gohma, K.; Komatsu, J.; Sekine, K.; Miyafuji, A.; Kunoh, J.; Honma, R.; Akita, Y.; Ohta, A. Heterocycles 1992, 33, 257. doi:10.3987/COM-91-S29

13. Okazawa, T.; Satoh, T.; Miura, M.; Nomura, M. J. Am. Chem. Soc. 2002, 124, 5286. doi:10.1021/ja0259279

14. Masui, K.; Ikegami, H.; Mori, A. J. Am. Chem. Soc. 2004, 126, 5074. doi:10.1021/ja031855p

15. Masui, K.; Mori, A.; Okano, K.; Takamura, K.; Kinoshita, M.; Ikeda, T. Org. Lett. 2004, 6, 2011. doi:10.1021/ol049386z

16. Amaladass, P.; Clement, J. A.; Mohanakrishnan, A. K. Tetrahedron 2007, 63, 10363. doi:10.1016/j.tet.2007.07.037

17. Turner, G. L.; Morris, J. A.; Greaney, M. F. Angew. Chem., Int. Ed. 2007, 46, 7996. doi:10.1002/anie.200702141

18. Arai, N.; Miyaoku, T.; Teruya, S.; Mori, A. Tetrahedron Lett. 2008, 49 , 1000. doi:10.1016/j.tetlet.2007.12.010

19. Roger, J.; Požgan, F.; Doucet, H. Green Chem. 2009, 11, 425. doi:10.1039/b819912d

20. Liégault, B.; Lapointe, D.; Caron, L.; Vlassova, A.; Fagnou, K. J. Org. Chem. 2009, 74, 1826. doi:10.1021/jo8026565

21. Zinovyeva, V. A.; Vorotyntsev, M. A.; Bezverkhyy, I.; Chaumont, D.; Hierso, J.-C. Adv. Funct. Mater. 2011, 21, 1064 doi:10.1002/adfm.201001912

22. Beydoun, K.; Roger, J.; Boixel, J.; Le Bozec, H.; Guerchais, V.; Doucet, H. Chem. Commun. 2012, 48, 11951. doi:10.1039/c2cc37046h

23. Glover, B.; Harvey, K. A.; Liu, B.; Sharp, M. J.; Tymoschenko, M. F. Org. Lett. 2003, 5, 301. doi:10.1021/ol027266q

24. Lavenot, L.; Gozzi, C.; Ilg, K.; Orlova, I.; Penalva, V.; Lemaire, M. J. Organomet. Chem. 1998, 567, 49. doi:10.1016/S0022-328X(98)00667-6

25. Fournier dit Chabert, J.; Marquez, B.; Neville, L.; Joucla, L.; Broussous, S.; Bouhours, P.; David, E.; Pellet-Rostaing, S.; Marquet, B.; Moreau, N.; Lemaire, M. Bioorg. Med. Chem. 2007, 15, 4482. doi:10.1016/j.bmc.2007.04.023

26. Forgione, P.; Brochu, M.-C.; St-Onge, M.; Thesen, K. H.; Bailey, M. D.; Bilodeau, F. J. Am. Chem. Soc. 2006, 128, 11350 doi:10.1021/ja063511f

27. Liégault, B.; Petrov, I.; Gorlesky, S. I.; Fagnou, K. J. Org. Chem. 2010, 75, 1047. doi:10.1021/jo902515z

28. Borghese, A.; Geldhof, G.; Antoine, L. Tetrahedron Lett. 2006, 47, 9249. doi:10.1016/j.tetlet.2006.10.130

29. Pierre, C.; Baudoin, O. Tetrahedron 2013, 69, 4473. doi:10.1016/j.tet.2012.11.060

30. René, O.; Fagnou, K. Org. Lett. 2010, 12, 2116. doi:10.1021/ol1006136

31. Dong, J. J.; Roy, D.; Roy, R. J.; Ionita, M.; Doucet, H. Synthesis 2011, 3530. doi:10.1055/s-0030-1260213

\section{License and Terms}

This is an Open Access article under the terms of the Creative Commons Attribution License

(http://creativecommons.org/licenses/by/2.0), which permits unrestricted use, distribution, and reproduction in any medium, provided the original work is properly cited.

The license is subject to the Beilstein Journal of Organic Chemistry terms and conditions:

(http://www.beilstein-journals.org/bjoc)

The definitive version of this article is the electronic one which can be found at:

doi:10.3762/bjoc. 10.123 